\title{
Student Research Abstract: Modeling the cerebellum, from neurons to healthy and pathological behaviors
}

\author{
Alberto Antonietti \\ Politecnico di Milano \\ Piazza Leonardo da Vinci 32 \\ 20133, Milano, Italy \\ +39-02-2399-9501 \\ alberto.antonietti@polimi.it
}

\section{PROBLEM AND MOTIVATION}

The human nervous system and its functioning have always been the focus of attention for many scientists for centuries and they were studied from many different points of view. This research project bridges neuroscience, neurocomputing and bioengineering and aims to explore the brain neural mechanisms with two approaches: modeling and experimental. Since the entire nervous system is too complex and wide to be studied as a whole, the research is focused on the cerebellum and its known crucial learning plasticity skills. The proposal has a strong multi-scale approach, from detailed neuron properties to motor behavior, connecting realistic modeling and human experimental data, with the perspective of useful clinical-rehabilitative applications.

\section{BACKGROUND AND RELATED WORK}

The cerebellum influences behavior via interactions with other brain structures. Different cerebellar regions play an integral role in the control of various behaviors including voluntary limb movements, eye movements, balance, locomotion, and even higher (cognitive) brain functions. It is also clear that the cerebellum exerts control over the flexibility of these behaviors; cerebellar integrity is critical for trial-and-error adaptation of motor behaviors to new contexts (motor learning) [1,7]. Due to its crucial role, cerebellar pathologies led to a considerable impairment in motor control and learning (e.g. ataxia, dysmetria, asynergia, abnormal temporal association, etc.). The impact of cerebellar disorders is being growingly recognized worldwide, but there is still a medical need to enhance the basic knowledge and to develop effective therapies. Many efforts have been put into building a comprehensive theory of learning focused on cerebellum, both by modeling in detail its structure and functionalities, and by experimentally testing its role in human subjects. It is crucial to define a causality chain linking plasticity mechanisms, neural circuits and behavioral learning. The critical position in brain circuitry and the involvement in sensorimotor and cognitive processing make the cerebellum an ideal structure

Permission to make digital or hard copies of part or all of this work for personal or classroom use is granted without fee provided that copies are not made or distributed for profit or commercial advantage and that copies bear this notice and the full citation on the first page. Copyrights for third-party components of this work must be honored. For all other uses, contact the Owner/Author.

Copyright is held by the owner/author(s).

SAC 2016, April 04-08, 2016, Pisa, Italy

ACM 978-1-4503-3739-7/16/04.

http://dx.doi.org/10.1145/2851613.2852017 for studying the possible role of neuronal plasticity, in a translational perspective, on learning [6].

Since the seventies, various scientists have been trying to build cerebellar computational models, the first one was proposed by Marr and Albus [1, 7] and from then to now models based on different approaches and scales were proposed. Starting from neurophysiological measures, such as patch-clamps on cerebellar neurons, single cells or small networks were modeled in detail with the Neuron software [9]. These models were highly realistic, capturing the biophysical properties of the neurons, but the simulations were computational time demanding. To simulate medium and large-scale networks of neurons, both analog models, which describe the evolution of the firing rates of the cells as continuous variables, and spiking neural networks (SNN) were exploited [3-5]. In particular, SNN were able to replicate the cerebellar topology by means of thousands Integrate\&Fire neurons, maintaining the biological fidelity (connection ratios, firing rates, synaptic plasticity, etc.) and allowing real-time performances on a standard desktop computer (Intel Core i7-2600 CPU@3.40 GHz).

\section{APPROACH AND UNIQUENESS}

The present research project will tackle three challenges, summarized in a schematic way in Figure 1.

\subsection{First Challenge: Healthy Models}

The first two challenges of the project regard the modeling of the cerebellum: the first one aims at generating realistic cerebellar models, using SNNs, testing their functionality in cerebellum-driven tasks both in simulation and embedding them into the controller of a robotic platform. We would obtain a realistic model of a healthy human cerebellum into an embodied system acting and sensing in real-world. In this way, the built neurorobot could show behaviors similar to healthy human subjects. The collaboration with neurophysiologists will allow us to insert the cutting-edge knowledge at level of neurons and network dynamics into realistic neural models.

\subsection{Second Challenge: Pathological Models}

The second challenge aims at modifying the developed "healthy model" to obtain specific "pathological models", starting from neurophysiological data and behavioral experiments on animals and humans with cerebellar lesions. Each cerebellar disease could have a corresponding pathological model where one or more functional mechanisms are corrupted. Then, we can test the functionality of different pathological models in simulations and 
in neurorobotic experiments to analyze the behavioral outcomes for different pathologies.

Manifold examples of cerebellar impairments can be found in literature and it is possible to translate these physiological damages into model's modifications. For example, cortical cerebellar degeneration decreases the volume of the cerebellar cortex, thus we can translate it decreasing the number of Purkinje cells in the model. Another example is the mutation of the gene that encodes the protein Myosin-Va, this mutation causes neurological diseases like Griscelli and Elejalde syndromes in humans. The Myosin-Va protein involves cerebellar learning, specifically Long Term Depression (LTD) at cortical level. This impairment can be translated by a decrease of the LTD parameter of the model's learning rule that governs the synaptic plasticity in the cerebellar cortex.

A realistic cerebellar model, able to generate human-like behaviors when embedded into embodied systems, is a precious tool for specific manipulations and thus promising in fostering our understanding about learning mechanisms, cerebellar deficits and potential treatment actions.

\subsection{Third Challenge: Clinical Applications}

In the third challenge, the models will be translated into a clinical context. We will design experimental tests that involve an interaction between the cerebellar patients and an ad-hoc robotic setup (haptic robot, electromyograph, eye tracker, etc.) in order to compare the behavioral results in these tests with the behavioral outcomes generated by the neurorobot controlled by the different cerebellar pathological models. In this way, the designed tests could provide in an absolutely non-invasive way a diagnostic hypothesis about the cerebellar pathology and the site of the cerebellar lesion of the patients.



Figure 1. Block diagram of the different project tasks.

The relevance of this research project is that, for the first time, a realistic modeling reconstruction of the cerebellum will be used to fit experimental datasets, collected from both healthy and pathological subjects, with the aim to unveil the implicit microcircuit computations of the network operating in closed-loop tasks. A possible further development of the project could be the challenging goal to simulate novel approaches of treatment and rehabilitation for cerebellar patients, using for instance visual feedback or Functional Electric Stimulation controlled by the neurorobot, which could drive the predictive/corrective learning usually carried out by the "intact" cerebellum.

\section{RESULTS AND CONTRIBUTIONS}

Starting from the state of the art in the field of computational models inspired to the cerebellum, refining an existing SNN [5], we validated the proposed cerebellar model in three neurophysiological tasks where the cerebellum is critically involved: the Eye Blink Classical Conditioning (EBCC), the vestibulo-ocular reflex and the reaching movement perturbed by force fields. We carried out both in-silico simulations and tests with the network embodied into a robotic platform [3]. Since the SNN has multiple free parameters (e.g. learning rule constants and initialization weights) that have to be tuned, we developed a metaheuristic technique for an automatic tuning. The Genetic Algorithm (GA) automatically found the SNN parameters that can reproduce desired behaviors [2]. We exploited the GA to find the model parameters that fitted the behavioral outcome of human subjects, which participated to a neurophysiological EBCC experiment [8]. The model was able to reproduce the human data, confirming physiological hypotheses about the influence of the Transcranial Magnetic Stimulation applied on the cerebellar cortex.

\section{REFERENCES}

[1] Albus, J.S. 1971. A theory of cerebellar function. Mathematical Biosciences. 10, 1-2 (Feb. 1971), 25-61.

[2] Antonietti, A. et al. 2015. Spiking Neural Network with Distributed Plasticity Reproduces Cerebellar Learning in Eye Blink Conditioning Paradigms. IEEE Transactions on Biomedical Engineering. (Oct. 2015).

[3] Casellato, C. et al. 2014. Adaptive Robotic Control Driven by a Versatile Spiking Cerebellar Network. PLOS ONE. 9, 11 (Nov. 2014), e112265.

[4] Casellato, C. et al. 2015. Distributed cerebellar plasticity implements generalized multiple - scale memory components in real - robot sensorimotor tasks. Frontiers in Computational Neuroscience. 9, (Feb. 2015).

[5] Luque, N.R. et al. 2011. Cerebellarlike corrective model inference engine for manipulation tasks. IEEE transactions on systems, man, and cybernetics. Part B, Cybernetics. 41, 5 (Oct. 2011), 1299-312.

[6] Markram, H. 2006. The blue brain project. Nature reviews. Neuroscience. 7, 2 (Feb. 2006), 153-60.

[7] Marr, D. 1969. A theory of cerebellar cortex. The Journal of physiology. 202, 2 (Jun. 1969), 437-70.

[8] Monaco, J. et al. 2014. Cerebellar theta burst stimulation dissociates memory components in eyeblink classical conditioning. The European journal of neuroscience. 40, July (Sep. 2014), 1-8.

[9] Solinas, S. et al. 2010. A realistic large-scale model of the cerebellum granular layer predicts circuit spatio-temporal filtering properties. Frontiers in cellular neuroscience. 4 , (Jan. 2010), 12. 